\title{
The Application of Mobile GIS Based on Wireless Sensor Network in the Forest Environment Real-time Monitoring
}

\author{
Lu Liao, Ting Bao \\ Beijing Engineering Research Center for IoT Software \\ and Systems \\ Beijing University of Technology, Beijing, China \\ E-mail: lulu2999@126.com, baoting@emails.bjut.edu.cn
}

\author{
Jin-Chao Ban \\ Remote Sensing and Mapping Division, Space Star \\ Technology CO., LTD., Beijing, China \\ E-mail: banjch503@163.com
}

\begin{abstract}
Accurate and reliable data collection and real-time dynamic monitoring are the basis for realizing the intelligent forest. Aiming at the problem of real-time dynamic environmental parameters monitoring in protected areas, a wireless sensor network (WSN) will be constructed, and ZigBee and mobile geographic information systems (GIS) technology will be combinedon iOS mobile development platform to design a WSN, which can be used in the field or in the room. It is an application which can display the forest environment parameters dynamically manage the WSN. This application can capture the user's location of the Geographic Information System (GPS) coordinates. It can also display with GIS and allow users to modify the data that obtained by the sensor. In this paper, the application can be used on the iOS operating system platform. It is developed with ArcGISrelated software and application programming interface (API).
\end{abstract}

Keywords-mobile GIS; forest environment; iOS; real-time dynamic monitoring; WSN

\section{INTRODUCTION}

Currently, with the smart of forestry requirements continue to increase, the information, networking and intelligent management and monitoring of forestry has become the prerequisite and basis for supervision of forest workers to adapt to the new opportunities and the new challenges. It is also an important means to accelerate the modern forestry construction. Mobile GIS is a set of GIS, GPS and mobile communications technology in one of the three major systems. The most prominent feature is the support of the mobile terminal, the user location in the process of changing, the collected information in a timely manner and presented to the user to meet the forest monitoring and protection of mobility and real-time requirements [1]. With the development of Internet of Things technology and the widespread use of mobile devices, people now can use sensor networks to measure the physical state of the environment.

Aiming at the problem of building smart forest, we design a system based on WSN and GIS technology including hardware and software. Its advantages are low cost, easy deployment, easy maintenance and real-time dynamic monitoring of forest ecological environment parameters. The system sensing layer will collect and supervise the forest eco-environmental parameters by using GPS, temperature, humidity, PM2.5, wind speed and wind direction sensors in real time. For the forest environment monitoring and management to provide a good technical means [2].

In the recent years, mobile devices are widely used by people. Smart phones have become the most popular devices for applications that are used successfully in various fields like shopping, map navigation, geology, education, agriculture and so on. Smart phones are believed to be a integration of conventional mobile phone and a personal digital assistant into a whole part. They have become an indispensable part of daily lives of people such as e-mail, information inquiry, news Browsing and internet surfing. With the increase in computing power of efficient processors, broadband Internet access and productivityenhancing applications, Smart phones and tablets have the ability to store information such as personal and laptop computers [3]. The use of Smart phones saves money by not having to buy separate equipment and facilitates faster access to information at anytime, anywhere.

Various platforms with different operating systems and various development tools can be used to develop applications for Smart phones and tablets. Among them, there are six different platform providers are believed as the primary forces in the Smart phone and tablets market. They are Google with the Android OS, Apple with the iOS, Nokia with the Symbian OS, Microsoft with the Windows CE OS family, RIM with the Blackberry OS and LiMo Foundation with the Linux Mobile [4]. However, with the passage of time and the development of science and technology, the main Smart phone and tablets operating system on the market at present is Android OS and Apple with the iOS [5]. Developers who need to develop their applications based on the various platforms are required to use the respective native Software Development Kit that provide related code samples, libraries, compilers, tools and so on. In the situation of Apple's iOS platform that we have used for developing this application in this study, Objective-C is the primary develop language. Registering as an iOS developer and downloading the Apple's iOS SDK are necessary [6].

In the few recent years, the development of Smart phones and tablets with touch screen display, WiFi, sensors, keyboards, multimedia recording capabilities and Global Positioning System has make people to continuously obtain, view and share geo-referenced contents. Furthermore, with the development in the area of computer 
and GIS technology, Smart phones and tablets can server as quick reference tools for people in many fields like environmental protection and geosciences with related applications. I have developed this application for Smart phones and tablets that assists geologists and related users with the collection or display of field data and data management [7].

Above all, the goal of this study is to develop a tool for collecting and displaying primary forest environment data covering the whole district area, and to design systems for analyzing this data. In this background, an iOS application has been devised for displaying relevant geographic and sensor information on iPad platform [8]. This application based on GIS, it can combine the visual effect of map and geographic analysis function to natural database operations. This application employs GPS, Cellular Mobile Network and Wi-Fi for positioning. The application has included Satellite image and Topographic map that enable users to choose suitable map between different conditions while they use this application [9]. In addition, other features are also comprised such as area measurements, distance, route and GPS results could be display on the equipments with ArcGIS software and relate development frameworks. The combination of the application with the devices' built-in GPS capabilities, allows for the collection of data incorporating coordinate and spatial information, with the ability to store, modify, update, search, manage, analyze, and report findings related to spatial information. The application can be used to support surveying, mapping and geosciences fieldworks for any area in the nature reserve [10].

\section{RELATED WORK}

\section{A. WSN}

Wireless sensor network is a distributed sensor network, composed of several hundreds to thousands of nodes which are capable of sensing, actuation, and the ability to communicate [11]. In our study, we use six different sensors, including temperature, humidity, PM2.5, negative oxygen icon, wind speed, wind direction. They can monitor forest environment parameters in a multidirectional and comprehensive way. This smart forest system consists of sensors, monitoring terminals, communications and ad hoc networks, GIS server and mobile display platform. The monitoring terminal module can collect the various environment parameters. The communication and ad hoc network module can realize the function of data transmission, which can send the sensors data to the server with $4 \mathrm{G}$ network technology. The GIS server and display platform can show the data stored in the server. Further more, based on the research of sensing technology in the forest environment monitoring system, we use sensor fusion technology to improve the accuracy of the data. Using data fusion technology, we can detect and synthesize the collaborative information of each monitoring terminal with multi-level, multi-aspect. The information of different sources, time and place is fused, finally can get the environmental data that is more accurate.

\section{B. Real-Time Mobile GIS}

Real-time GIS means the system should complete the specified action in time, which including real-time operating system and real-time database. Forest environmental monitoring system is a real-time system, because the data collected by each sensor node in the system is real-time and dynamic data. Therefore, it is necessary to collect, process and analyze the data in time. Forest environment monitoring system has higher requirements on data processing, storage capacity and transmission speed [12].

The following will explain how to solve the GIS technology embedded in the forest environment, and finally realize the real-time and dynamic display on iPad platform. First, it is necessary to optimize the efficiency of the map service display, the map service is divided into a base map and a feature layer. In order to ensure sufficient refresh efficiency, and reduce the conversion of map coordinates to screen coordinates frequency, base map and thematic layers with different strategies in the maintenance and display [13]. When creating a map service, we should separate them into different two parts. Put the no real-time information on the part of the base map, slice processing is necessary before publish it as map services, and then cache it as a picture that storage on the server. The dynamic maps that change often should be placed on the feature layer. In consideration of the speed about real-time data updates and display, we should create at least two different map documents on ArcGIS Online, and finally in the client's map display overlay two map service layer. When the client loads, drawing the base map, and then draw the dynamic feature layer. When the information in the dynamic feature layer changes, it only needs to refresh the screen icons of these change points and keep the information of the other elements unchanged. This will improve the efficiency of the whole real-time refresh procedure.

\section{The Publishing of Various Maps}

A GIS lets us visualize, question, analyze, and interpret data to understand relationships, patterns, and trends. GIS benefits organizations of all sizes and in almost every industry. There is a growing interest in and awareness of the economic and strategic value of GIS.

Through pre-research, this experiment will process and publish a variety of map on ArcGIS related platform. ArcGIS is a complete set of GIS platform products which is researched and developed by Esri with over forty years consulting experience. The system has powerful map making, spatial data management, spatial analysis, spatial information integration, publishing and sharing abilities. ArcGIS can not only provide innovative desktop GIS applications, configurable server portals, ready-to-use Apps, more application development options for users but also can provide strong support for building the next generation of Web GIS applications. 
ArcGIS Online is a complete, cloud-based mapping platform. ArcGIS Online allows users to publish GIS web services to an Esri-administered cloud environment [14]. Users don't have to install anything; they just sign in to their ArcGIS Online account. There are two types of services they can deploy:

- Feature services expose the geometry, attributes, and symbol information for vector GIS features. They are appropriate for displaying, querying, and editing your business data on top of web basemaps.

- Tiled map services expose a set of pregenerated map images (known as a map cache) that can be viewed as basemaps in a web mapping application. When you publish your service, you can also ask the server to create and store a cache of tiles. You can then bring the tiles into your web map by accessing the service's URL.

Using a combination of tiled map services and feature services in your application allows fast mapping while supporting query and editing operations.

ArcGIS Online hosted services are available through organizational subscriptions to ArcGIS Online. An advantage of using ArcGIS Online is that you don't have to install any server software or tune the services. The services run in an Esri-administered cloud environment in which the server automatically scales up to meet demand.

In the field, users often work in areas without a data connection. If that's the case, users can download the map to make it available when they do not have a connection. They can also download or copy basemaps directly to the device and reuse them across multiple maps. Once they have the map offline, they can work with the map, including collecting and editing data, in the same way as when the network connected.

Firstly, we should prepare maps on ArcGIS Online platform. Developer should author the map for offline use allows data collectors to view, collect, and update features when disconnected from the Internet. Once reconnected, data collectors can synchronize with the map, send any updates they have, and get map updates from other collectors. When creating or saving a map that meets the offline requirements on ArcGIS Online, offline mode is automatically enabled. For maps that meet the offline requirements but that existed before offline mode, enable the offline mode as follows:

- Find the map in My Content.

- Go to the item details of the map and click Edit to edit its item properties.

- In the Properties section, choose Enable offline mode.

- If one would like to specify which types of information field workers download and sync, choose Show advanced options. See Optimize download and sync for details.

To author a map for offline use, the map needs to contain the following: Sync-enabled feature layers-These can be hosted feature layers or ArcGIS Server feature services. Feature layers that are not sync-enabled are not supported.

\section{SYSTEM DESIGN}

\section{A. Physical Structure Model of the System}

"3G" technology including the satellite Remote Sensing (RS), GIS and GPS. Nowadays, " $3 G$ " technology is widely used in monitoring forest environment in many countries. It can realize the rapid collection and processing of forest environment information. In this paper, we combine with " $3 \mathrm{G}$ " technology based on Field-Programmable Gate Array (FPGA) platform to form a comprehensive forest health monitoring system. The system build a forest monitoring experimental platform composed of six different kinds of sensors (wind speed, wind direction, PM2.5, negative oxygen ions and temperature and humidity sensors), FPGA and communication modules ( $4 \mathrm{G}$ and ZigBee) are used, which combine with ArcGIS related software and API, and finally display real-time GIS data on iPad. We design a complete set of forest health monitoring system for data acquisition, processing, transmission and related application.

The whole system is divided into two parts, the system sensing layer and the system application layer. The system sensing layer, which is the hardware system of real-time dynamic monitoring of the wireless sensing network. It uses the ZigBee based wireless ad hoc network technology and GPRS wireless transmission technology to locate the relevant data in the forest environment in real-time. The system application layer is based on C/S architecture, which can dynamically collect, monitor and analyze the spatial distribution and dynamic of environmental parameters on the basis of the effective management of the nodes in the forest environment WSN. It can deal with emergencies in time. The topology structure of the mobile GIS system is shown as below:

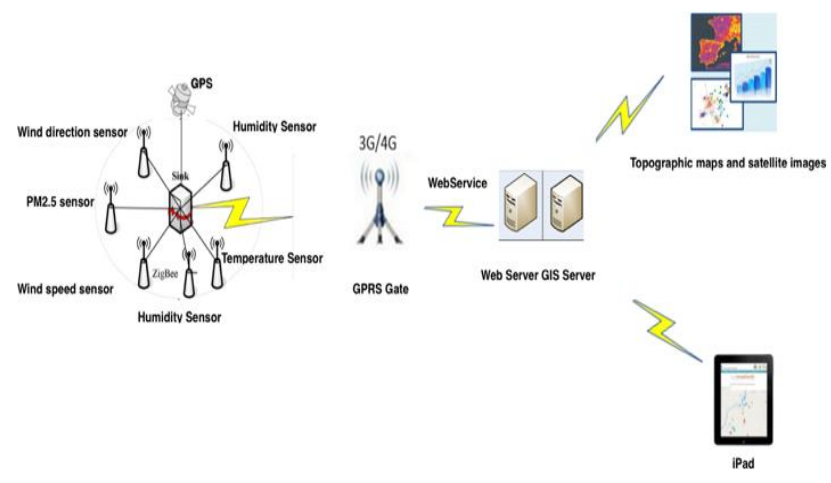

Figure 1. The topology structure of the mobile GIS system

\section{B. Application Components}

This application can provide three different services. Each service corresponds to each geospatial service, which are: the download, display and process services. The first download service is to allow users to download geospatial data, especially sensor data. The second service is to allow the visualization of the sensors data. The last service is to process the data, with the ability to perform other operations using the data acquired by the sensors [15]. 


\section{1) Make a map}

This application opens a web mapon ArcGIS Online which displays data of various sensors call. The web map also contains popup definitions that describe how information about the various sensors should be displayed. When users tap on an icon, the application uses AGSPopupsContainerViewController to display popup information about that feature in a callout. The popup allows edits to attributes, attachments, or geometry of the feature, and the edits are committed to the service using an AGSFeatureLayer and AGSAttachmentManager.

Each individual's data, with the same GPS location, will be grouped together and display as a single pin on ArcGIS Online as shown in below.

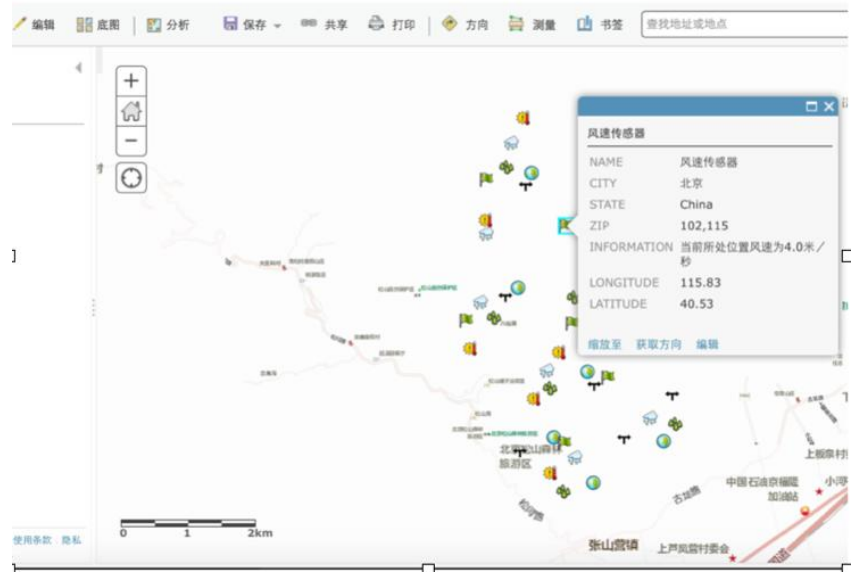

Figure 2. Sensors located on a map with ArcGIS Online platform

\section{2) Datainput}

The sensor data was collected along with the GPS location information, including latitude and longitude coordinates obtained from the device hardware, to identify the location of each sensor. Each sensor location was plotted on ArcGIS Online Maps.

\begin{tabular}{ll}
\hline Sensors & Push pin \\
\hline Wind speed sensor & Wind direction sensor \\
Temperature sensor & Humidity sensor \\
PM2.5 sensor & Negative oxygen ion sensor
\end{tabular}

Figure 3. Different style icons of various sensors

\section{3) Graphic user interface(GUI)}

This application allows users to edit geographic data in an ArcGIS Feature service. Users can add new features with photo/video as attachment(s), or they can edit and delete existing features.

- Tap on any of the incidents displayed on the map.

- Tap on the callout accessory button to view details in a popup and make edits.

- Tap on the action button in the top right, then choose "Edit". Notice that popup shifts into edit mode. Tap on any of the attributes to make changes.

- Tap on the Geometry or Attachment icons at the bottom of the popup to make changes to the feature's shape or edit attachments.

- Tap on "Done" in the popup to save your edits.

- Back on the map display, you can tap the "+" button in the top right to add a new feature. This displays a template picker. Choose a type of incident to add. A popup will display.

- Specify attribute values for the feature.

- Tap on the Geometry icon on the bottom of the popup to specify where to add the feature to the map. Hit "Done" and save your edits.

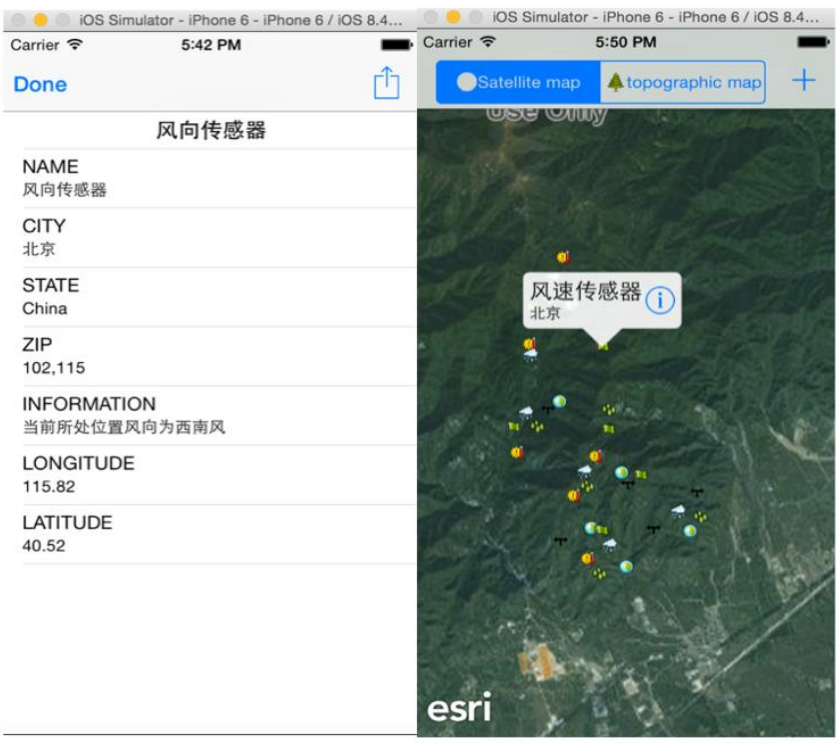

Figure 4. GUI of sensors information on iPad

\section{CONCLUSION}

With the rapid development of information technology, this paper puts forward a system of real-time dynamic monitoring technology in forest environment, aiming at the need of building a smart forest. System uses Zigbee wireless ad hoc network technology research and develop the system sensing layer of WSN, which realize the forest environment related parameters of real-time dynamic collection and transmission with low cost. The data transmission between the system sensing layer and the application layer is realized by GPRS technology, which is 
used to collect and transfer the real-time data to the system application layer.

At the same time, as the latest advancement in the Smart phone or tablets technology and operation systems have given a far more diverse functionality. The applications are designed and developed based on operation systems that can be installed on many Smart phone or tablets models to provide them with various extra functions. In this study, I have successfully developed the this application based on iPad mobile client. One of the main benefits of using the Apple's iOS is that it is quiet easy to develop applications using the Apple's SDK. Under the premise of using the ArcGIS software and related API, the application can display and share spatial data on iOS mobile client. It can be more intuitive to show the quality of the environment in the forest in the region, the degree of pollution and development trends. The system is flexible, easy to layout, easy to maintain, which can increase the handling capacity of emergency, and improve the ability of the forest environment supervision department to ensure the safety of the forest protection area.

\section{ACKNOWLEDGMENT}

The authors would also like to express appreciations to colleagues in our laboratory for their valuable comments and other helps.

\section{REFERENCES}

[1] Apple Inc. 2012. iOS technology overview. Cupertino, CA: Apple.

[2] K. Katevas, H. Haddadi, and L. Tokarchuk, "Poster: Sensingkit: A multi-platform mobile sensing framework for large-scale experiments," in Proceedings of the 20th Annual International Conference on Mobile Computing and Networking, ser. MobiCom '14. New York, NY, USA: ACM, 2014, pp. 375-378. [Online]. Available: http://doi.acm.org/10.1145/2639108.2642910.

[3] A. Bröring, A. Remke, and D. Lasnia, "SenseBox -A generic sensor platform for the web of things," in Mobile and Ubiquitous Systems:
Computing, Networking, and Services (Lecture Notes of the Institute for Computer Sciences, Social Informatics and Telecommunications Engineering), vol. 104. Berlin, Germany: Springer-Verlag, 2012, ch. 16, pp.186-196.

[4] H. Haddadi, F. Ofli, Y. Mejova, I. Weber, and J. Srivas- tava, "360degree quantified self," in Healthcare Informatics (ICHI), 2015 International Conference on, Oct 2015, pp. 587-592.

[5] Apple Inc. 2012. iOS technology overview. Cupertino, CA: Apple Inc.

[6] L. Huang, Y. Xu, X. Chen, H. Li, and Y. Wu, "Design and Implementation of Location Based Mobile Health System," in 2012 Fourth International Conference on Computational and Information Sciences, 2012, pp. 919-922.

[7] Jonoski A, Alfonso L, Almoradie A, Popescu I, van Andel SJ, Vojinovic Z. 2012. Mobile phone applications in the water domain. Environ Eng Manage J. 11:919-930.

[8] Monares A, Ochoa SF, Pino JA, Herskovic V, Rodriguez-Covili J, Neyem A. 2011. Mobile computing in urban emergency situations improving the support to firefighters in the field. Expert Syst Appl. 38:1255-1267.

[9] Halvorsen O, Clarke D. 2011. Mac OS X and iOS. In: Halvorsen O, Clarke D, editors. OS X and iOS Kernel programming. New York, NY: Apress; p. 15-38.

[10] M. Rouached, S. Baccar, and M. Abid, "RESTful sensor web enablement services for wireless sensor networks," in Proc. IEEE 8th World Congr. Services, Jun. 2012, pp. 65-72.

[11] P Kinney,"ZigBee Technology: Wireless Control that Simply. Works", IEEE Communications Design Conference, Oct 2003.

[12] S. Jirka, D. Nüst, and B. Proß, "Sensor Web and Web Processing Standards for Crisis Management," presented at the 10th International Conference on Information Systems for Crisis Response and Management, Baden-Baden, Germany, May 2013.

[13] Application of Real Time GIS using Remote Sensing and RTK-GPS for Local Government, Matsuda Naoko, Resume of Master's thesis Open Hearing Conference at KIT, (2004) .

[14] ArcGISOnline.Available:http://www.esri.com/software/arcgis/arcgis online[Aeecssed:07-12-2016] .

[15] ArcGIS Runtime SDK for iOS.Available:https://developers.arcgis. com/ios/.[Accessed:08-12-2016] . 